\title{
Theory of optical transitions in curved chromophores
}

William Barford, and Max Marcus

Citation: The Journal of Chemical Physics 145, 124111 (2016);

View online: https://doi.org/10.1063/1.4962747

View Table of Contents: http://aip.scitation.org/toc/jcp/145/12

Published by the American Institute of Physics

\section{Articles you may be interested in}

Theory of optical transitions in $\pi$-conjugated macrocycles

The Journal of Chemical Physics 144, 154102 (2016); 10.1063/1.4946794

Theory of optical transitions in conjugated polymers. II. Real systems

The Journal of Chemical Physics 141, 164102 (2014); 10.1063/1.4897985

Theory of optical transitions in conjugated polymers. I. Ideal systems

The Journal of Chemical Physics 141, 164101 (2014); 10.1063/1.4897984

Perspective: Optical spectroscopy in $\pi$-conjugated polymers and how it can be used to determine multiscale polymer structures

The Journal of Chemical Physics 146, 130902 (2017); 10.1063/1.4979495

Theory of exciton transfer and diffusion in conjugated polymers

The Journal of Chemical Physics 141, 164103 (2014); 10.1063/1.4897986

Intrachain exciton dynamics in conjugated polymer chains in solution

The Journal of Chemical Physics 143, 084102 (2015); 10.1063/1.4929378

\section{AIP | The Joumal of Chemical Physics}




\title{
Theory of optical transitions in curved chromophores
}

\author{
William Barford ${ }^{1}$ and Max Marcus ${ }^{1,2}$ \\ ${ }^{1}$ Physical and Theoretical Chemistry Laboratory, Department of Chemistry, University of Oxford, \\ Oxford OX1 3QZ, United Kingdom \\ ${ }^{2}$ Magdalen College, University of Oxford, Oxford OX1 4AU, United Kingdom
}

(Received 22 July 2016; accepted 1 September 2016; published online 28 September 2016)

\begin{abstract}
Using first order perturbation theory in the Born-Oppenheimer regime of the Frenkel-Holstein model, we develop a theory for the optical transitions in curved chromophores of $\pi$-conjugated polymers. Our key results are that for absorption, $A$, and emission, $I$, polarized parallel to the $0-0$ transition, $I^{01} / I^{00} \simeq A^{01} / A^{00}=S(N)$, where $S(N)=S(1) /$ IPR is the effective Huang-Rhys parameter for a chromophore of $N$ monomers and IPR is the inverse participation ratio. In contrast, absorption and emission polarized perpendicular to the $0-0$ transition acquires vibronic intensity via the Herzberg-Teller effect. This intensity generally increases as the curvature increases and consequently $I^{01} / I^{00}$ increases (where $I^{01}$ is the total $0-1$ emission intensity). This effect is enhanced for long chromophores and in the anti-adiabatic regime. We show via DMRG calculations that this theory works well in the adiabatic regime relevant to $\pi$-conjugated polymers, i.e., $\hbar \omega /|J| \lesssim 0.2$. Published by AIP Publishing. [http://dx.doi.org/10.1063/1.4962747]
\end{abstract}

\section{INTRODUCTION}

As shown by Spano and co-workers, ${ }^{1,2}$ the emissive vibronic spectrum of linear $\mathrm{J}$-aggregates and $\pi$-conjugated polymers reveals information about the exciton coherence length. In particular, these authors showed that the emission intensity ratio satisfies $I^{01} / I^{00}=S(1) / N$, where $S(1)$ is the Huang-Rhys parameter for a single monomer which forms the aggregate or polymer and $N$ is the exciton coherence length. In related work, Barford and co-workers ${ }^{3,4}$ showed that within the Born-Oppenheimer approximation both for emission and absorption the intensity ratios satisfy $I^{01} / I^{00} \simeq A^{01} / A^{00}$ $=S(1) / \mathrm{IPR}$, where IPR (the exciton inverse participation ratio) can be regarded as the average chromophore size in conformationally disordered $\pi$-conjugated polymers. From an experimental perspective, these results are useful as they allow the chromophore sizes to be determined, and in principle polymer conformations to be found. ${ }^{5}$

Hestand and Spano $^{6}$ subsequently showed that the prediction $I^{01} / I^{00}=S(1) / N$ fails in bent polymers; a result also confirmed in Refs. 4 and 7 . The origin of this failure is essentially the Herzberg-Teller effect, which means that the $0-1$ transition acquires additional intensity from states that are optically forbidden in linear systems.

This work extends Hestand and Spano's analysis ${ }^{6}$ to investigate the absorption and emission intensities for curved chromophores. Since we assume open boundary conditions for the exciton wavefunctions, we derive explicit expressions for the longitudinal and transverse polarized transitions. We show that for the $0-1$ transition polarized parallel to the $0-0$ transition, the expressions $I^{01} / I^{00} \simeq A^{01} / A^{00}=S(1) /$ IPR are still valid. Further, we show how in general the expression $I^{01} / I^{00}=S(1) /$ IPR fails as a function of chromophore size, curvature, and adiabaticity.

Since our focus is optical transitions in $\pi$-conjugated polymers, we solve the Frenkel-Holstein model in the Born-
Oppenheimer regime. We use perturbation theory to develop a theory of the vibronic progression and we use density matrix renormalization (DMRG) calculations to check this theory.

\section{THE FRENKEL-HOLSTEIN MODEL}

The Frenkel-Holstein model has been motivated previously as a coarse-grained model that describes both the delocalization of the Frenkel exciton and its coupling to local normal modes (or Einstein oscillators) in $\pi$-conjugated polymers. ${ }^{2,3,8,9}$

We define the Frenkel-Holstein model as

$$
H_{\mathrm{FH}}=H_{\mathrm{ex}}+H_{\mathrm{ph}}+H_{\mathrm{ep}} .
$$

The exciton term is

$$
H_{\mathrm{ex}}=\Delta \sum_{n=1}^{N} a_{n}^{\dagger} a_{n}+J \sum_{n=1}^{N-1}\left(a_{n+1}^{\dagger} a_{n}+a_{n}^{\dagger} a_{n+1}\right),
$$

where $\hat{a}_{n}^{\dagger}$ creates a Frenkel exciton on the $n$th monomer and $\Delta$ is the exciton excitation energy on monomer $n . J(<0)$ is the nearest-neighbor exciton transfer integral, which includes both through-space and through-bond contributions. ${ }^{9}$ The phonon term is

$$
H_{\mathrm{ph}}=\hbar \omega \sum_{n=1}^{N} b_{n}^{\dagger} b_{n},
$$

where $b_{n}^{\dagger}$ creates an excitation in the Einstein oscillator on monomer $n$ and $\omega$ is its angular frequency. Finally, the excitonphonon coupling term is

$$
H_{\mathrm{ep}}=-g \hbar \omega \sum_{n=1}^{N} a_{n}^{\dagger} a_{n}\left(b_{n}^{\dagger}+b_{n}\right),
$$

where $g$ (also denoted as $\lambda$ in Ref. 1 or $A / \sqrt{2}$ in Ref. 3) is the exciton-phonon coupling constant. The Huang-Rhys 
parameter for a single monomer is $S(1)=g^{2}$. Typical FrenkelHolstein model parameters for $\pi$-conjugated polymers ${ }^{4}$ are $|J| \sim 2 \mathrm{eV}, \hbar \omega \sim 0.2 \mathrm{eV}$, and $g \sim 1-2$.

For a system with open boundary conditions, the Bloch transforms

$$
\tilde{a}_{j}^{\dagger}=\left(\frac{2}{N+1}\right)^{1 / 2} \sum_{n=1}^{N} \sin \left(\frac{n \pi j}{N+1}\right) a_{n}^{\dagger}
$$

and

$$
\tilde{b}_{k}^{\dagger}=\left(\frac{2}{N+1}\right)^{1 / 2} \sum_{n=1}^{N} \sin \left(\frac{n \pi k}{N+1}\right) b_{n}^{\dagger}
$$

diagonalize $H_{\mathrm{ex}}$ and $H_{\mathrm{ph}}$. Inserting the inverses of Eqs. (5) and (6) into Eqs. (2) and (3), and using orthonormality gives

$$
H_{\mathrm{ex}}+H_{\mathrm{ph}}=\sum_{j=1}^{N}\left(\Delta+2 J \cos \left(\frac{\pi j}{N+1}\right)\right) \tilde{a}_{j}^{\dagger} \tilde{a}_{j}+\hbar \omega \sum_{j=1}^{N} \tilde{b}_{j}^{\dagger} \tilde{b}_{j} .
$$

Similarly, Eq. (4) becomes

$$
H_{\mathrm{ep}}=-g \hbar \omega\left(\frac{2}{N+1}\right)^{3 / 2} \sum_{i j k} V(i, j, k) \tilde{a}_{i}^{\dagger} \tilde{a}_{j}\left(\tilde{b}_{k}^{\dagger}+\tilde{b}_{k}\right),
$$

where

$$
\begin{aligned}
V(i, j, k)= & \frac{1}{4}\left[\cot \left(\frac{\pi(i+j-k)}{2(N+1)}\right)+\cot \left(\frac{\pi(j+k-i)}{2(N+1)}\right)\right. \\
& \left.+\cot \left(\frac{\pi(k+i-j)}{2(N+1)}\right)-\cot \left(\frac{\pi(i+j+k)}{2(N+1)}\right)\right] .
\end{aligned}
$$

$V(i, j, k)$ has the property that if $(i+j) \in$ even then $V(i, j, k)$ $=0$ for $k \in$ even, while if $(i+j) \in$ odd then $V(i, j, k)=0$ for $k \in$ odd.

We now treat $\left(H_{\mathrm{ex}}+H_{\mathrm{ph}}\right)$ as the unperturbed part of the full Hamiltonian, and denote it as $H_{0}$, while $H_{\mathrm{ep}}$ is the perturbation, and is denoted as $H_{1} . H_{0}$ has the exact (unperturbed) Born-Oppenheimer eigenstates,

$$
\left|j ; n_{k_{1}}, n_{k_{2}}, \ldots, n_{k_{N}}\right\rangle^{(0)}=|j\rangle \otimes\left|n_{k_{1}}, n_{k_{2}}, \ldots, n_{k_{N}}\right\rangle,,
$$

where

$$
\begin{gathered}
|j\rangle=\tilde{a}_{j}^{\dagger}|0\rangle, \\
\left.\left.\left|n_{k}\right\rangle\right\rangle=\frac{\left(\tilde{b}_{k}^{\dagger}\right)^{n_{k}}}{\sqrt{n_{k} !}}|0\rangle\right\rangle,
\end{gathered}
$$

and $|0\rangle$ and $|0\rangle\rangle$ are the vacuums for the exciton and phonon Hilbert spaces, respectively. The unperturbed eigenenergy is $E^{(0)}\left(j ; n_{k_{1}}, n_{k_{2}}, \ldots, n_{k_{N}}\right)=\Delta+2 J \cos \left(\frac{\pi j}{N+1}\right)+\hbar \omega \sum_{k=1}^{N} n_{k}$.

\section{0-0 ABSORPTION}

The $0-0$ transition dipole moment associated with the $j$ th exciton state is

$$
\underline{\mu}_{j}^{00}={ }^{(0)}\left\langle j ; n_{k}=0|\underline{\hat{\mu}}| \mathrm{GS} ; 0\right\rangle,
$$

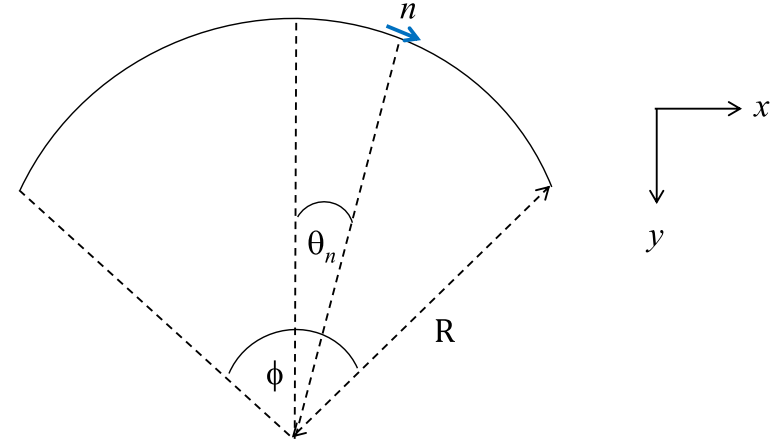

FIG. 1. A regular arc representing a curved chromophore in a twodimensional plane of $N$ monomers subtending an angle $\phi$ with a radius of curvature $R$. The transition dipole moment of the $n$th monomer is $\mu_{0}\left(\cos \theta_{n}, \sin \theta_{n}\right)$.

where

$$
|\mathrm{GS} ; 0\rangle=|0\rangle \otimes|0\rangle\rangle .
$$

Assuming a curved chromophore in the $x-y$ plane, the transition dipole operator is

$$
\underline{\hat{\mu}}=\mu_{0} \sum_{n=1}^{N}\left(\cos \theta_{n}, \sin \theta_{n}\right)\left(a_{n}+a_{n}^{\dagger}\right),
$$

where $\theta_{n}$ is defined in Fig. 1 . Thus, using

$$
\left.\left|j ; n_{k}=0\right\rangle^{(0)}=\tilde{a}_{j}^{\dagger}|0\rangle \otimes|0\rangle\right\rangle,
$$

we obtain

$$
\mu_{x, j}^{00}= \begin{cases}0 ; & j \in \text { even } \\ \mu_{0} \sum_{n} \Psi_{n}^{j} \cos \theta_{n} ; & j \in \text { odd }\end{cases}
$$

and

$$
\mu_{y, j}^{00}=\left\{\begin{array}{ll}
\mu_{0} \sum_{n} \Psi_{n}^{j} \sin \theta_{n} ; & j \in \text { even } \\
0 ; & j \in \text { odd }
\end{array},\right.
$$

where the exciton center-of-mass wavefunction is

$$
\Psi_{n}^{j}=\left(\frac{2}{N+1}\right)^{1 / 2} \sin \left(\frac{n \pi j}{N+1}\right) .
$$

For large $N$,

$\mu_{x, j}^{00}=\left\{\begin{array}{ll}0 ; & j \in \text { even } \\ \mu_{0}(2 N)^{1 / 2}\left(\frac{\sin (\alpha / 2)}{\alpha}+\frac{\sin (\beta / 2)}{\beta}\right) ; & j \in \text { odd }\end{array}\right.$,

$\mu_{y, j}^{00}=\left\{\begin{array}{ll}\mu_{0}(2 N)^{1 / 2}\left(\frac{\sin (\alpha / 2)}{\alpha}-\frac{\sin (\beta / 2)}{\beta}\right) ; & j \in \text { even } \\ 0 ; & j \in \text { odd }\end{array}\right.$,

and

$$
\alpha=(j \pi+\phi),
$$

while

$$
\beta=(j \pi-\phi) .
$$

As the $j=1$ and $j=2$ states dominate the optical intensity for curved chromophores, we neglect higher 


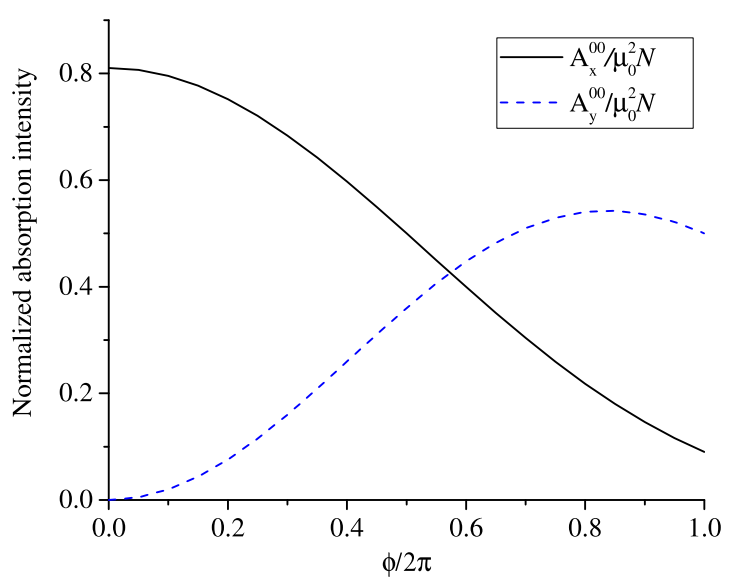

FIG. 2. The normalized $x$ and $y$ polarized absorption intensities, $A_{x}^{00}$ from the $j=1$ exciton state and $A_{y}^{00}$ from the $j=2$ exciton state (see Eq. (20)), as a function of the angle subtended by the chromophore, $\phi$, as defined in Fig. 1. Notice that $A_{x}^{00}$ does not vanish at $\phi=2 \pi$ because of open boundary conditions. Assuming Kasha's rule (i.e., emission from the $j=1$ state), the emission intensities satisfy $I_{x}^{00} \propto A_{x}^{00}$ and $I_{y}^{00}=0$.

energy $j$ states and now define the $x$ and $y$ polarized $0-0$ absorption intensities as (ignoring multiplicative constants)

$$
A_{x}^{00}=\left(\mu_{x, j=1}^{00}\right)^{2}
$$

and

$$
A_{y}^{00}=\left(\mu_{y, j=2}^{00}\right)^{2} .
$$

These intensities are plotted in Fig. 2 as a function of chromophore curvature.

\section{0-1 ABSORPTION}

Since the transition dipole operator only contains exciton creation and annihilation operators, and not phonon operators, (see Eq. (16)), the matrix element ${ }^{(0)}\left\langle j ; n_{k}=1|\hat{\mu}| \mathrm{GS} ; 0\right\rangle$ vanishes. Thus, the $0-1$ absorption intensity arises by mixing of $\left|j ; n_{k}=1\right\rangle^{(0)}$ with $\left|j^{\prime} ; n_{k}=0\right\rangle^{(0)}$ via the perturbation $H_{1}$. In particular, to first-order in perturbation theory

$$
\begin{aligned}
\left|j ; n_{k}=1\right\rangle \simeq & \left|j ; n_{k}=1\right\rangle^{(0)} \\
& -\sum_{j^{\prime}} \frac{(0)\left\langle j^{\prime} ; n_{k}=0\left|\hat{H}_{1}\right| j, n_{k}=1\right\rangle^{(0)}}{\Delta E_{j^{\prime} ; j, k}^{(0)}}\left|j^{\prime} ; n_{k}=0\right\rangle^{(0)},
\end{aligned}
$$

where

$$
\Delta E_{j^{\prime} ; j, k}^{(0)}=E^{(0)}\left(j^{\prime} ; n_{k}=0\right)-E^{(0)}\left(j ; n_{k}=1\right) .
$$

\section{A. $x$-polarized absorption to the $j=1$ state}

As Fig. 3 illustrates, $x$-polarized $0-1$ absorption to the $j=1$ state occurs by mixing of $\left|j=1 ; n_{k}=1\right\rangle^{(0)}$ with $\left|j=1 ; n_{k}=0\right\rangle^{(0)}$ via $H_{1}$. (In this analysis, mixing with higher energy odd $-j$ states is neglected.) Since

$$
\begin{aligned}
\langle j= & \left.1 ; n_{k}=0\left|\hat{H}_{1}\right| j=1, n_{k}=1\right\rangle^{(0)} \\
& =-g \hbar \omega\left(\frac{2}{N+1}\right)^{3 / 2} V(1,1, k)
\end{aligned}
$$

and

$$
\Delta E_{1 ; 1, k}^{(0)}=-\hbar \omega
$$

we obtain

$$
\begin{aligned}
\left|j=1 ; n_{k}=1\right\rangle \simeq & \left|j=1 ; n_{k}=1\right\rangle^{(0)}-g\left(\frac{2}{N+1}\right)^{3 / 2} \\
& \times V(1,1, k)\left|j=1 ; n_{k}=0\right\rangle^{(0)}
\end{aligned}
$$

for odd $k$.

Thus, the $x$-component of the $0-1$ transition dipole moment associated with the $k$ th phonon mode is

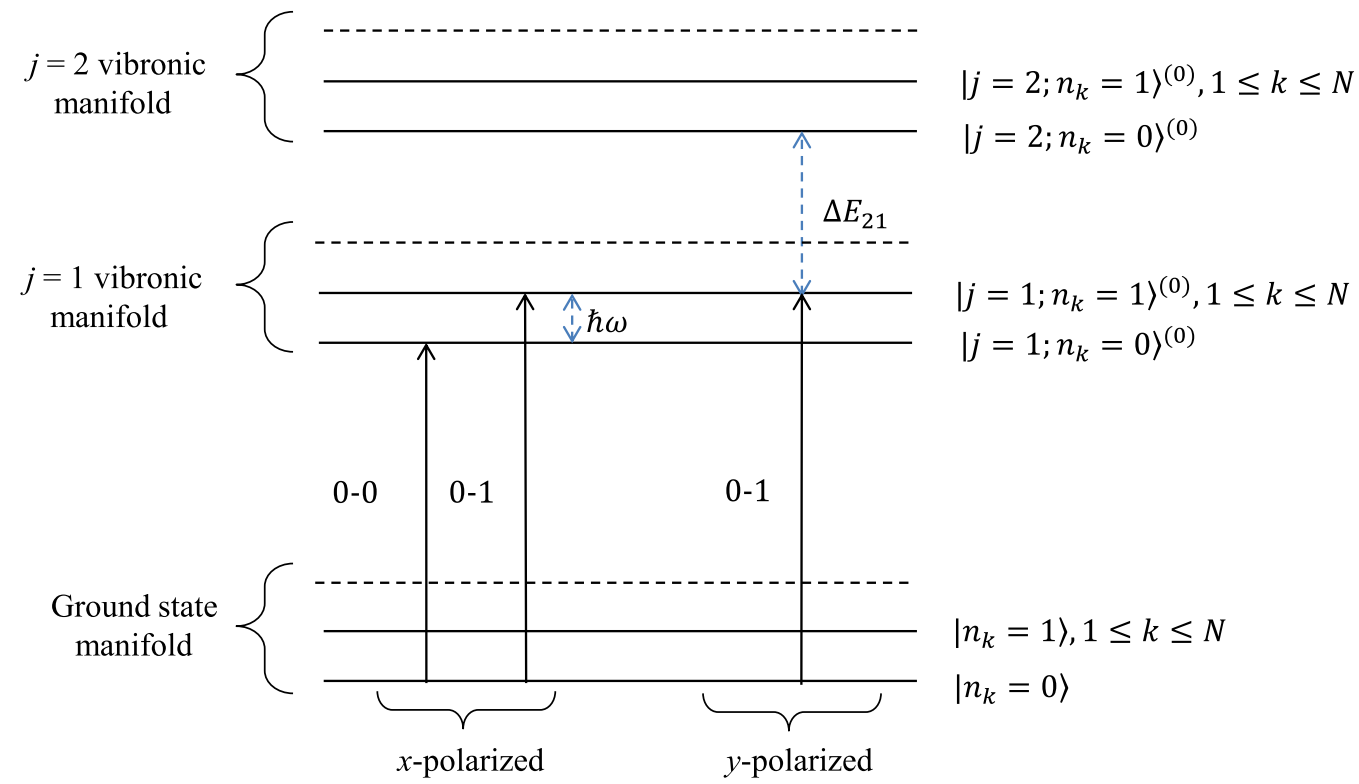

FIG. 3. The $0-0$ and $0-1$ absorptions to the $j=1$ vibronic manifold. The $x$ and $y$ polarized $0-1$ absorption occurs via mixing of $\left|j=1 ; n_{k}=1\right\rangle^{(0)}$ with $\left|j=1 ; n_{k}=0\right\rangle^{(0)}$ and $\left|j=2 ; n_{k}=0\right\rangle^{(0)}$, respectively (as indicated by the blue-dashed arrows). The $x$ and $y$ polarizations are defined in Fig. 1 . 
$\mu_{x, j=1}^{01}(k)=\left\langle j=1 ; n_{k}=1|\hat{\mu}| \mathrm{GS} ; 0\right\rangle$

$$
= \begin{cases}0 ; & k \in \text { even, } \\ -\mu_{x, 1}^{00} \times g\left(\frac{2}{N+1}\right)^{3 / 2} V(1,1, k) ; & k \in \text { odd, }\end{cases}
$$

where $\mu_{x, j}^{00}$ is defined in Eq. (18). The total $x$-polarized $0-1$ absorption intensity is obtained by summing over all phonon modes,

$$
\begin{aligned}
A_{x}^{01} & =\sum_{k \in \text { odd }}\left(\mu_{x, 1}^{01}(k)\right)^{2} \\
& =A_{x}^{00} \times g^{2}\left(\frac{2}{N+1}\right)^{3} \sum_{k \in \text { odd }} V^{2}(1,1, k),
\end{aligned}
$$

where $A_{x}^{00}$ is defined in Eq. (25).

We have shown numerically that

$$
\begin{aligned}
\left(\frac{2}{N+1}\right)^{3} \sum_{k \in \text { odd }} V^{2}(1,1, k) & =\frac{3}{2(N+1)} \\
& \equiv \frac{1}{\text { IPR }},
\end{aligned}
$$

where IPR is the inverse participation ratio for a chromophore of size $N$, defined by

$$
\operatorname{IPR}=\frac{1}{\sum_{n}\left|\Psi_{n}\right|^{4}} .
$$

For the standing-wave solutions ${ }^{3,10}$ (Eq. (20)),

$$
\mathrm{IPR}=2(N+1) / 3 .
$$

Thus we find

$$
A_{x}^{01}=A_{x}^{00} \frac{g^{2}}{\operatorname{IPR}}
$$

or

$$
\frac{A_{x}^{01}}{A_{x}^{00}}=S(N)
$$

where

$$
S(N)=\frac{g^{2}}{\mathrm{IPR}}
$$

is the effective Huang-Rhys parameter for a chromophore of size $N$.

Equation (38) is precisely the result obtained for linear chromophores in Ref. 3 using the Born-Oppenheimer approximation where the phonons were treated classically; it was also validated by DMRG calculations.

\section{B. $y$-polarized absorption to the $j=1$ state}

As Fig. 3 illustrates, $y$-polarized $0-1$ absorption to the $j=1$ state occurs by mixing of $\left|j=1 ; n_{k}=1\right\rangle^{(0)}$ with $\left|j=2 ; n_{k}=0\right\rangle^{(0)}$ via $H_{1}$. (Again, mixing with higher energy even- $j$ states is neglected.) This acquired intensity, polarized orthogonally to the $0-0$ intensity, is analogous to the HerzbergTeller effect: The exciton-phonon interaction has mixed a vibronic state (i.e., $\left|j=1 ; n_{k \in \text { even }}=1\right\rangle^{(0)}$ ) with the same spatial symmetry as a pure exciton state (i.e., $\left.\left|j=2 ; n_{k}=0\right\rangle^{(0)}\right)$. ${ }^{11}$
Following the same procedure as in Section IV A we obtain

$$
\begin{aligned}
\left|j=1 ; n_{k}=1\right\rangle \simeq & \left|j=1 ; n_{k}=1\right\rangle^{(0)}+\frac{g \hbar \omega}{\Delta E_{21}^{(0)}}\left(\frac{2}{N+1}\right)^{3 / 2} \\
& \times V(2,1, k)\left|j=2 ; n_{k}=0\right\rangle^{(0)}
\end{aligned}
$$

for even $k$, where

$$
\begin{aligned}
\Delta E_{21}^{(0)} & =E^{(0)}\left(j=2 ; n_{k}=0\right)-E^{(0)}\left(j=1 ; n_{k}=1\right) \\
& =2|J|\left[\cos \left(\frac{\pi}{N+1}\right)-\cos \left(\frac{2 \pi}{N+1}\right)\right]-\hbar \omega .
\end{aligned}
$$

Thus, the $y$-polarized $0-1$ absorption to the $j=1$ vibronic manifold is

$$
A_{y}^{01}=A_{y}^{00} g^{2}\left(\frac{\hbar \omega}{\Delta E_{21}^{(0)}}\right)^{2}\left(\frac{2}{N+1}\right)^{3} \sum_{k \in \text { even }} V^{2}(2,1, k),
$$

where $A_{y}^{00}$ is defined in Eq. (26).

Again we find numerically that,

$$
\begin{aligned}
\left(\frac{2}{N+1}\right)^{3} \sum_{k \in \text { even }} V^{2}(2,1, k) & =\frac{1}{(N+1)} \\
& \equiv \frac{2}{3 \times \text { IPR }} .
\end{aligned}
$$

Thus,

$$
\frac{A_{y}^{01}}{A_{y}^{00}}=\frac{2 S(N)}{3}\left(\frac{\hbar \omega}{\Delta E_{21}^{(0)}}\right)^{2}
$$

\section{0-1 EMISSION}

Assuming Kasha's rule, emission occurs from the lowestenergy dipole-allowed excited state. To zeroth order, this state is $\left|j=1 ; n_{k}=0\right\rangle^{(0)}$, which has a non-vanishing transition dipole moment only to $|G S ; 0\rangle$. Emission to excited vibrational levels in the ground state manifold occurs by mixing of $\left|j=1 ; n_{k}=0\right\rangle^{(0)}$ with $\left|j ;\left\{n_{k}\right\}\right\rangle^{(0)}$ via $H_{1}$. In particular, the $0-1$ emission occurs to

$$
\left.\left|\mathrm{GS} ; n_{k}=1\right\rangle=|0\rangle \otimes\left|n_{k}=1\right\rangle\right\rangle ; \quad \forall k .
$$

Thus, following Section IV, we require the perturbed state,

$$
\begin{aligned}
\left|j=1 ; n_{k}=0\right\rangle \simeq & \left|j=1 ; n_{k}=0\right\rangle^{(0)} \\
& -\sum_{j^{\prime}} \frac{{ }^{(0)}\left\langle j^{\prime} ; n_{k}=1\left|\hat{H}_{1}\right| j=1 ; n_{k}=0\right\rangle^{(0)}}{\Delta E_{j^{\prime}, k ; j}^{(0)}} \\
& \times\left|j^{\prime} ; n_{k}=1\right\rangle^{(0)} .
\end{aligned}
$$

\section{A. $x$-polarized emission from the $j=1$ state}

As Fig. 4 illustrates, the $x$-polarized $0-1$ emission occurs via mixing of $\left|j=1 ; n_{k}=0\right\rangle^{(0)}$ with $\left|j=1 ; n_{k}=1\right\rangle^{(0)}$. Thus,

$$
\begin{aligned}
\left|j=1 ; n_{k}=0\right\rangle \simeq & \left|j=1 ; n_{k}=0\right\rangle^{(0)}+g\left(\frac{2}{N+1}\right)^{3 / 2} \\
& \times V(1,1, k)\left|j=1 ; n_{k}=1\right\rangle^{(0)}
\end{aligned}
$$




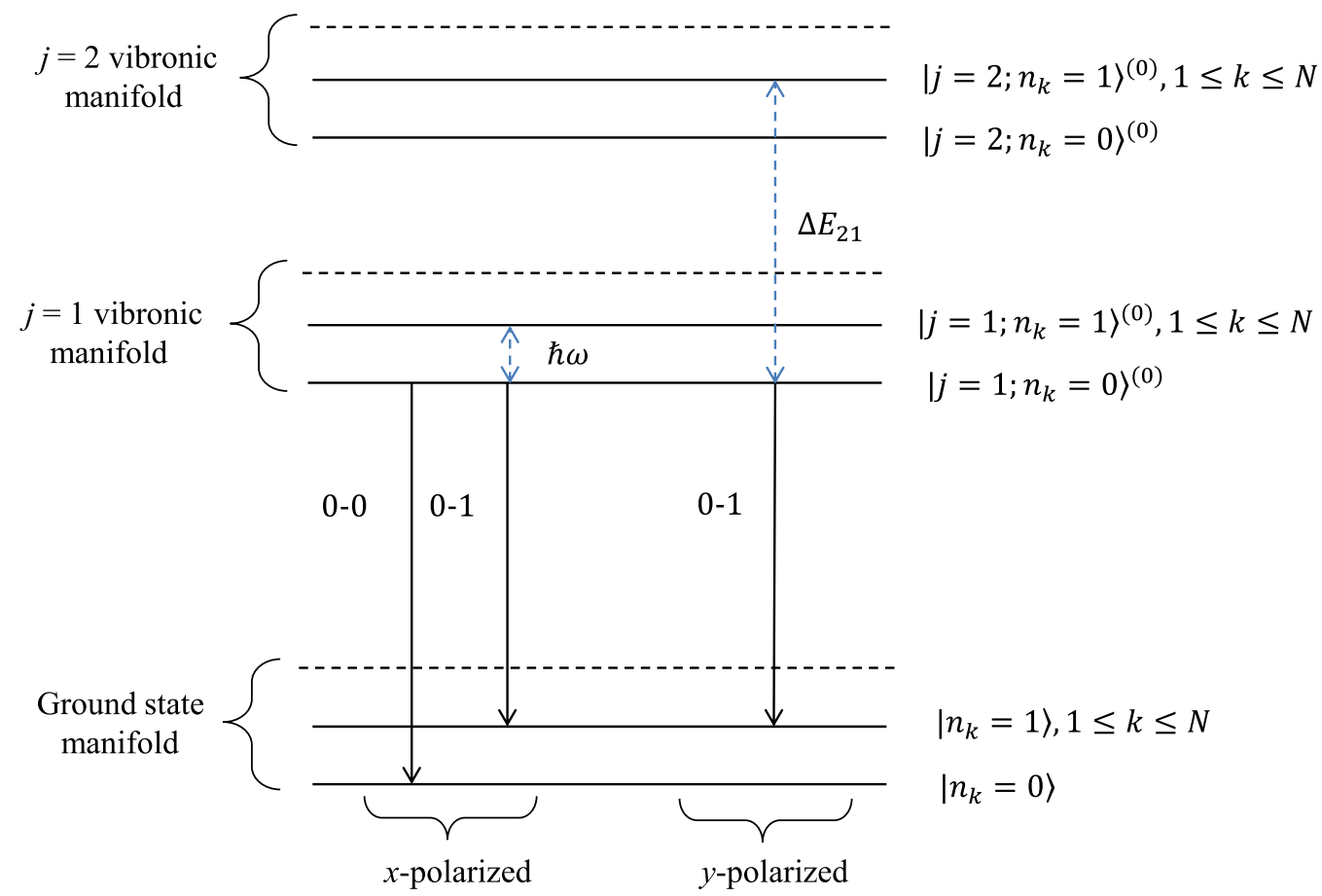

FIG. 4. The $0-0$ and $0-1$ emissions from the $j=1$ exciton ground state. The $x$ and $y$ polarized $0-1$ emission occurs via mixing of $\left|j=1 ; n_{k}=0\right\rangle^{(0)}$ with $\left|j=1 ; n_{k}=1\right\rangle^{(0)}$ and $\left|j=2 ; n_{k}=1\right\rangle^{(0)}$, respectively (as indicated by the blue-dashed arrows).

for odd $k$. The emission transition dipole moment is

$$
\begin{aligned}
\mu_{x, j=1}^{01}(k) & =\left\langle j=1 ; n_{k}=0|\hat{\mu}| \mathrm{GS} ; n_{k}=1\right\rangle \\
& = \begin{cases}0 ; & k \in \text { even }, \\
\mu_{x, 1}^{00} \times g\left(\frac{2}{N+1}\right)^{3 / 2} V(1,1, k) ; & k \in \text { odd } .\end{cases}
\end{aligned}
$$

Thus, as for Section IV A,

$$
\begin{aligned}
\left(\mu_{x, 1}^{01}\right)^{2} & =\sum_{k \in \text { odd }}\left(\mu_{x, 1}^{01}(k)\right)^{2} \\
& =\left(\mu_{x, 1}^{00}\right)^{2} \times S(N),
\end{aligned}
$$

which implies that

$$
\begin{aligned}
I_{x}^{01} & =I_{x}^{00} \times S(N) \\
& \equiv I^{00} \times S(N)
\end{aligned}
$$

(if we assume that $\hbar \omega \ll E^{(0)}\left(j=1 ; n_{k}=0\right)$ ).

An analogous expression to Eq. (50) was derived by Spano and co-workers ${ }^{1,2}$ and by Barford and Marcus ${ }^{3}$ for linear chromophores. Its utility for linear chromophores is that it means that the chromophore size can be determined, provided that $S(1)=g^{2}$ is known. The result encapsulated by Eq. (50) is particularly useful for curved chromophores, as it indicates that the intensity ratios in the longitudinal direction (i.e., parallel to the $0-0$ transition) also reveals the chromophore size.

\section{B. $y$-polarized emission from the $j=1$ state}

As Fig. 4 illustrates, the $y$-polarized emission occurs via mixing of $\left|j=1 ; n_{k}=0\right\rangle^{(0)}$ with $\left|2=1 ; n_{k}=1\right\rangle^{(0)}$.
Using analogous methods to Sections IV B and V A, we find that

$$
\begin{aligned}
\left(\mu_{y, j=1}^{01}\right)^{2} & =\sum_{k \in \text { even }}\left(\mu_{y, j=1}^{01}(k)\right)^{2} \\
& =\left(\mu_{y, j=2}^{00}\right)^{2} \frac{2 S(N)}{3}\left(\frac{\hbar \omega}{\Delta E_{21}^{(0)}}\right)^{2},
\end{aligned}
$$

where (in contrast to Eq. (41))

$$
\Delta E_{21}^{(0)}=2|J|\left[\cos \left(\frac{\pi}{N+1}\right)-\cos \left(\frac{2 \pi}{N+1}\right)\right]+\hbar \omega .
$$

\section{Summary}

We now use the results of the previous two sections to derive an expression for the intensity ratio $I^{00} / I^{01}$. Defining

$$
\begin{aligned}
I^{00} \equiv I_{x}^{00} & =\left(E_{j=1}\right)^{3}\left(\mu_{x, j=1}^{00}\right)^{2} \\
& =\left(E_{j=1}\right)^{2} A_{x}^{00}, \\
I_{x}^{01} & =\left(E_{j=1}\right)^{3}\left(\mu_{x, j=1}^{01}\right)^{2}, \\
I_{y}^{01} & =\left(E_{j=1}\right)^{3}\left(\mu_{y, j=1}^{01}\right)^{2},
\end{aligned}
$$

and

$$
I^{01}=I_{x}^{01}+I_{y}^{01}
$$

we obtain

$$
\frac{I^{00}}{I^{01}} \times S(N)=\frac{1}{\left(1+\frac{2}{3}\left(\frac{A_{y}^{00}}{A_{x}^{00}}\right)\left(\frac{\hbar \omega}{\Delta E_{21}^{(0)}}\right)^{2}\right)},
$$

where $S(N), A_{x}^{00}, A_{y}^{00}$, and $\Delta E_{21}^{(0)}$ are defined by Eqs. (39), (25), (26), and (52), respectively. 
Equation (57) has the following limits:

(1) In the adiabatic limit, i.e., $\hbar \omega /|J| \rightarrow 0$, then $\hbar \omega / \Delta E_{21}^{(0)}$ $\rightarrow 0$ and thus ${ }^{13}$

$$
\frac{I^{00}}{I^{01}} \times S(N) \rightarrow 1 .
$$

(2) In the antiadiabatic limit, i.e., $\hbar \omega /|J| \rightarrow \infty$ (which is generally more applicable to molecular J-aggregates), or the large chromophore limit, i.e., $N \rightarrow \infty$, then $\hbar \omega / \Delta E_{21}^{(0)} \rightarrow 1$ and thus

$$
\frac{I^{00}}{I^{01}} \times S(N) \rightarrow \frac{3 A_{x}^{00}}{\left(3 A_{x}^{00}+2 A_{y}^{00}\right)}
$$

We note that our result in this limit differs quantitatively from Ref. 6 because of our use of open boundary conditions.

Equations (58) and (59) are plotted in Fig. 5. Qualitatively, the reduction in the intensity ratio as a function of curvature can be understood via Fig. 2. $A_{x}^{00}$ decreases monotonically as the chromophore becomes more curved (but it does not vanish at $\phi=2 \pi$ because of the use of open boundary conditions). In contrast, except for $\phi \sim 2 \pi, A_{y}^{00}$ increases as the curvature increases. Since, from the Herzberg-Teller effect, $I_{01}$ acquires intensity from the $j=2, y$-polarized state, $I^{00} / I^{01}$ consequently decreases as the curvature increases.

The crossover between the limits (1) and (2) above occurs approximately when the chromophore size, $N$, satisfies

$$
N>\sqrt{3 \pi^{2}|J| / \hbar \omega} .
$$

Taking $\hbar \omega /|J|=0.1$ gives a value of $N \sim 20$; a result confirmed by Fig. 5 which shows that the $N=20$ calculation deviates more from the adiabatic limit (1) than the $N=10$ calculation.

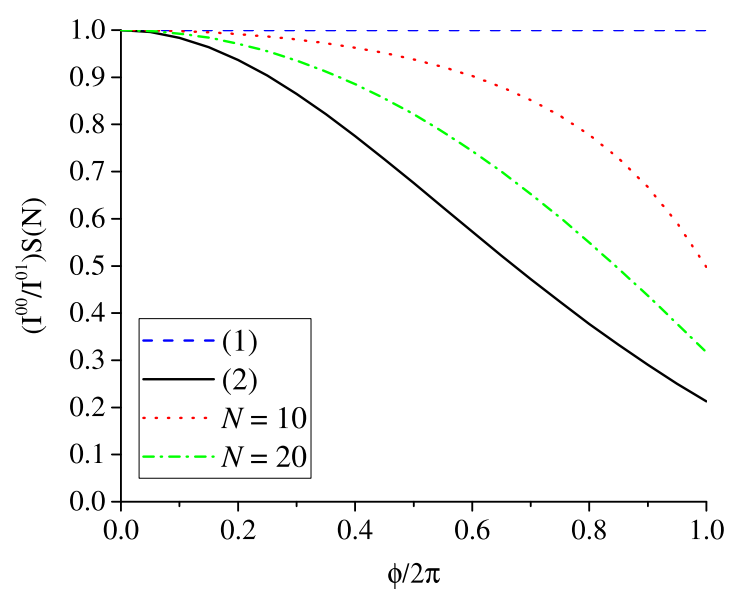

FIG. 5. The limiting cases of Eq. (57), $\left(I^{00} / I^{01}\right) \times S(N)$ versus $\phi$ (as defined in Fig. 1). (1) The adiabatic limit, $\hbar \omega /|J| \rightarrow 0$, Eq. (58), (dashed, blue line). (2) The anti-adiabatic limit $(\hbar \omega /|J| \rightarrow \infty)$ or large chromophores $(N \rightarrow \infty)$, Eq. (59) (solid, black curve). Also shown is Eq. (57) when $\hbar \omega /|J|=0.1$ for $N=10$ (dotted, red curve) and $N=20$ (dash-dotted, green curve).

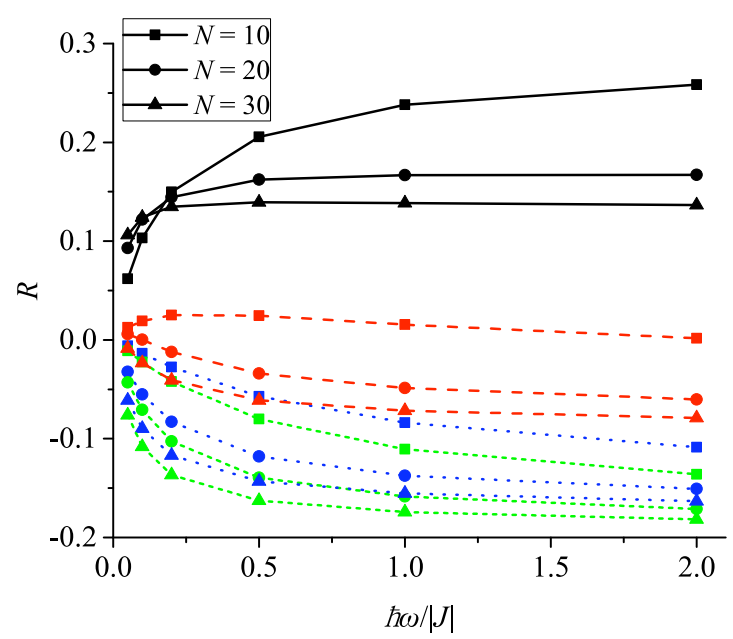

FIG. 6. The relative error, $R$ (Eq. (62)), between theory and DMRG calculations for $g=1 . \phi=3 \pi / 4$ (solid, black curves); $\phi=\pi / 2$ (dashed, red curves); $\phi=\pi / 4$ (dotted, blue curves); $\phi=0$ (short-dashed, green curves), where $\phi$ is defined in Fig. 1.

\section{DMRG Calculations}

We check our results from perturbation theory in the Born-Oppenheimer regime against essentially exact DMRG calculations. ${ }^{12}$ (The application of DMRG to the FrenkelHolstein model is discussed in detail in Refs. 3 and 7). If

$$
X \equiv \frac{I^{00}}{I^{01}} \times S(N)
$$

then we define the relative error between theory and the DMRG calculations as

$$
R=2 \frac{\left(X_{\text {theory }}-X_{\mathrm{dmrg}}\right)}{\left(X_{\text {theory }}+X_{\mathrm{dmrg}}\right)} .
$$

Fig. 6 shows the relative error versus $\hbar \omega /|J|$ for various chromophore sizes and curvature. Evidently, the theory works well in the adiabatic limit (and for values of $\hbar \omega /|J|$ that are relevant to conjugated polymers, i.e., $\hbar \omega /|J| \simeq 0.1$ ). It works less well for larger values of $\hbar \omega /|J|$. For larger values of $\hbar \omega /|J|$, we find a breakdown of the result obtained above, especially for strongly curved $(\phi \geq 1.5 \pi)$ and essentially straight $(\phi<0.5 \pi)$ chromophores. With increasing chain length we see better agreement for strongly curved chromophores, while for essentially straight ones the results agree less well. This is due to a reduced curvature for longer chains, in agreement with the general trend for short chains.

In additional calculations it was also found that the quality of the theoretical result is only a weak function of the excitonphonon coupling constant, $g$. It was found that for large $g$ more curved chromophores are better described, whereas for small $g$ more straight chromophores are accounted for more accurately. However, the differences are negligible, especially for the regime relevant to conjugated polymers (i.e., $g \approx 1$ ).

\section{CONCLUDING REMARKS}

Using first order perturbation theory in the BornOppenheimer regime of the Frenkel-Holstein model, we have developed a theory for the optical transitions in curved 
chromophores of $\pi$-conjugated polymers. Our key results are as follows:

1. For absorption and emission polarized parallel to the 0-0 transition, $I^{01} / I^{00} \simeq A^{01} / A^{00}=S(N)$, where $S(N)$ $=S(1) / \mathrm{IPR}$ is the effective Huang-Rhys parameter for a chromophore of $N$ monomers and IPR is the inverse participation ratio. This is precisely the same result obtained for a linear chain in the Born-Oppenheimer limit. ${ }^{3}$

2. In contrast, absorption and emission polarized perpendicular to the $0-0$ transition acquires vibronic intensity via the Herzberg-Teller effect. This intensity generally increases as the curvature increases and consequently $I^{01} / I^{00}$ increases (where $I^{01}$ is the total 0-1 emission intensity). This effect is enhanced for long chromophores and in the anti-adiabatic regime.

We showed via DMRG calculations that this theory works well in the adiabatic limit, i.e., $\hbar \omega /|J| \lesssim 0.2$.

This paper has considered uniform, curved chromophores. In practice, in an actual conformationally disordered linear or cyclic polymer, the polymer is segmented into chromophores, defined by the Anderson-localized local exciton ground states (LEGSs). ${ }^{4,7}$ The theory developed here also applies to these curved chromophores, where the IPR is recognized as the chromophore size. In a polymer film, however, the chromophore dipoles will have random orientations, so the average $0-1$ emission intensity parallel to the average $0-0$ emission intensity will have both parallel and perpendicular components from individual chromophores, implying that, in general, Eq. (50) cannot be used to extract the average chromophore size. As Fig. 5 indicates, however, for realistic parameters for conjugated polymers (i.e., $\hbar \omega /|J|=0.1^{4}$ ) the deviation of $\left(I^{00} / I^{01}\right) S(N)$ from unity is only appreciable for chromophore sizes $\gtrsim 20$ monomers with significant curvature, i.e., $\phi \gtrsim \pi$. Thus, in practice, we anticipate that the intensity ratio can be used to extract the average chromophore size in realistic polymer systems with reasonable accuracy.

Finally, we observe that the results presented in Fig. 5 assume a fixed chromophore size as a function of curvature.
In practice, however, if the chromophore size in a conjugated polymer is determined by Anderson localization of the exciton center-of-mass wavefunction (i.e., the exciton coherence length), then as the curvature increases the chromophore size will decrease. This is because the through-space contribution to the exciton transfer integral decreases in magnitude as neighboring monomers become misaligned, implying that the exciton band width decreases and hence the Andersonlocalization length (i.e., the IPR) decreases ${ }^{5}$ (see Eq. (33) of Ref. 4). In summary, as a portion of conjugated polymer becomes more curved there is a tendency for $I^{00} / I^{01}$ to decrease, because of the explanation given at the end of Section V C. This effect is enhanced by a tendency for the chromophore size to decrease, which — all other things being equal — also decreases $I^{00} / I^{01}$.

\section{ACKNOWLEDGMENTS}

M.M. gratefully acknowledges the award of a Zvi and Ofra Meitar Magdalen Graduate Scholarship.

${ }^{1}$ F. C. Spano, J. Chem. Phys. 122, 234701 (2005).

${ }^{2}$ F. C. Spano and H. Yamagata, J. Phys. Chem. B 115, 5133 (2011).

${ }^{3}$ W. Barford and M. Marcus, J. Chem. Phys. 141, 164101 (2014).

${ }^{4}$ M. Marcus, O. R. Tozer, and W. Barford, J. Chem. Phys. 141, 164102 (2014).

${ }^{5}$ T. Adachi, J. Vogelsand, and J. M. Lupton, J. Phys. Chem. Lett. 5, 2165 (2014).

${ }^{6}$ N. J. Hestand and F. C. Spano, J. Phys. Chem. B 118, 8352 (2014).

${ }^{7}$ M. Marcus, J. Coonjobeeharry, and W. Barford, J. Chem. Phys. 144, 154102 (2016).

${ }^{8}$ H. Yamagata and F. C. Spano, J. Chem. Phys. 135, 054906 (2011).

${ }^{9}$ W. Barford, J. Phys. Chem. A 117, 2665 (2013).

${ }^{10}$ R. Chang, J. H. Hsu, W. S. Fann, K. K. Liang, C. H. Chang, M. Hayashi, J. Yu, S. H. Lin, E. C. Chang, K. R. Chuang, and S. A. Chen, Chem. Phys. Lett. 317, 142 (2000).

${ }^{11}$ For a circular chromophore (with periodic boundary conditions) the totally symmetric state (denoted as $j=0$ ) is dipole forbidden and acquires vibronic intensity by Herzberg-Teller coupling to the totally non-symmetric dipole-allowed $j= \pm 1$ states. See Appendix A of Ref. 7 for further details.

${ }^{12}$ S. R. White, Phys. Rev. Lett. 69, 2863 (1992); Phys. Rev. B 48, 10345 (1993).

${ }^{13}$ This result is also reproduced in the adiabatic limit of Eqs. (S.9) and (S.10) of the supplementary information of Ref. 6 . 